\title{
Financial Modeling and Option Theory with the Truncated Levy Process
}

\author{
Andrew Matacz* \\ School of Mathematics and Statistics \\ University of Sydney, 2006 \\ Australia \\ Report 97-28, October 1997
}

\begin{abstract}
In recent studies the truncated Levy process (TLP) has been shown to be very promising for the modeling of financial dynamics. In contrast to the Levy process, the TLP has finite moments and can account for both the previously observed excess kurtosis at short timescales, along with the slow convergence to Gaussian at longer timescales. I further test the truncated Levy paradigm using high frequency data from the Australian All Ordinaries share market index. I then consider, for the early Levy dominated regime, the issue of option hedging for two different hedging strategies that are in some sense optimal. These are compared with the usual delta hedging approach and found to differ significantly. I also derive the natural generalization of the BlackScholes option pricing formula when the underlying security is modeled by a geometric TLP. This generalization would not be possible without the truncation.
\end{abstract}

\footnotetext{
*Email: andrewm@maths.usyd.edu.au
} 


\section{Introduction}

It has been widely appreciated for some time that fluctuations in financial data show consistent excess kurtosis indicating the presence of large fluctuations not predicted by Gaussian models. With the continuing growth in the derivatives industry, and the recent emphasis on better risk management practices, the need for models that can describe these large events has never been greater.

Several authors [1] have explored the stable Levy class of distributions as a possible alternative to the Gaussian. Levy distributions exhibit scaling or fractal properties which occur commonly in complex systems often studied in statistical physics. Indeed the Levy distribution does seem to provide a consistently better representation of financial data than the Gaussian distribution. Despite this it would be fair to say that there has been a lack of interest in this class of models, especially in the option theory literature (see [2] for some exceptions). One reason for this would have to be a reluctance to accept the infinite variance which is typical in these models. This makes it difficult to find an appropriate generalization of the firmly entrenched Black-Scholes option pricing framework. Perhaps a more important reason is that financial data tends to become more Gaussian over longer timescales [3, 4]. This property is also evident in the decay of the implied volatility smile obtained from observed option prices with increasing maturity [5]. These properties cannot be explained by Levy distributions due to their stable additive property (the central limit theorem does not apply due to their infinite variance).

A much more popular approach to explaining the excess kurtosis is based on the observation that the variance (or volatility in financial language) of financial data appears to behave randomly. Stochastic volatility will generate kurtosis in an otherwise Gaussian process and this has led to a body of literature that attempts to model volatility as a diffusion process (for a review see [5]). An important reason for the popularity of this approach is that it is still based on a Gaussian framework making it a relatively Black-Scholes friendly explanation for excess kurtosis. A major failing of stochastic volatility models is that they do not describe the ubiquitous power law or scaling properties observed in financial data. These properties are observed in the volatility correlation function [6], the PDF of high frequency price increments [4, 7], and the temporal decay of the peak of the PDF describing the financial process [4]. These last two properties are in fact, for short time horizons, well described by a simple Levy process.

The growing empirical evidence of power law properties in financial data has generated renewed interest in the Levy paradigm. Recent work has shown that the problems associated with the Levy distribution can be simply overcome by what is known as a truncated Levy distribution (TLD). The TLD is Levy like in the central part of the distribution, but has a cutoff in the far tails that is faster than the Levy power law tails. The cutoff will ensure the variance of the TLD is finite. Financial prices over time can be described with the truncated Levy flight (TLF) or its continuous time limit the truncated Levy process (TLP). The TLF is constructed from sums of independent and identically distributed random variables described by a TLD. Since the TLD has finite variance, the central limit theorem applies and the TLF 
approachs a Gaussian distribution as desired. However what is interesting is the existence of a characteristic timescale separating the Levy and Gaussian regimes. This timescale can be arbitrarily long due to the stable nature of the Levy distribution. Mantegna and Stanley [8] were the first to make the above observations regarding the TLF. They drew their conclusions based on the sums of independent Levy distributed random variables with a discrete cutoff in the tails. Inspired by these results, Koponen [9] derived an analytical form for the characteristic function of a TLD with an exponential cutoff in the tails. These theoretical results have led to several recent empirical studies all supporting the TLF as a simple and effective model of financial data 4, 7].

The accurate modeling of financial price series is important for the pricing and hedging of financial derivatives such as options. Research on option theory with alternative pricing models has tended to focus on the pricing issue. It is now well known that non-Gaussian pricing models lead to the familiar volatility smile effect caused by the 'fat' tails of the nonGaussian PDF's. These effects are well known will not be the focus here. What is much less understood and discussed is the issue of option hedging for non-Gaussian models. This is surprising because this would seem in many cases to be a more important issue than pricing. For liquid options (usually the vanilla types discussed here), the price will be determined by the market. The real use of the model is to define a hedging strategy. The models option price simply provides a way of testing the model against the market price. The standard approach to option pricing and hedging is the Black-Scholes framework. What is remarkable about the Black-Scholes case is that, for Gaussian or log-Gaussian pricing models, there exists a hedging strategy which will eliminate all the risk to an option seller. This leads to the well known delta hedging result which says that the hedge value is given by the derivative of the option price with respect to the current price of the underlying security. However for more general pricing models a riskless hedge does not exist and in these cases the Black-Scholes framework does not tell us how to proceed. This is especially problematic for times close to expiry where deviations from Gaussian are large. The standard approach is to simply apply the delta hedging procedure regardless of the pricing model used. However this approach is purely ad hoc as it has no clear theoretical basis.

The inadequacies of the Black-Scholes framework led Bouchaud and Sornette to develop a simple and more general approach to option theory [10, 11]. Although in general a riskless hedge does not exist, it is possible to find an optimal hedging strategy that will minimize some appropriate measure of risk. An obvious choice for a risk measure is the variance of the wealth distribution of an option seller (or its 4th moment which would place more weight on the tails). Bouchaud and Sornette derived an expression for this optimal trading strategy which is valid for any non-Gaussian pricing model. In general the optimal trading strategy is not given by the delta hedge, though it is recovered for the special case of Gaussian or log-Gaussian models.

The outline of this paper is as follows. In section 2 we will further test the TLP model using high frequency data from the Australian All Ordinaries share market index. With the derived parameter values for the TLP, we compare in section 3.1 the Bouchaud-Sornette optimal hedging strategy, the TLP delta hedging strategy and the Gaussian delta hedging 
strategy. Although the TLP has finite variance, in the early Levy dominated regime a tail distribution based method to find an optimal trading strategy may be preferable to a moment based method. With this in mind, in section 3.2 we adapt to the TLP a simple tail distribution based method used by Bouchaud et-al [2, 11] to find the optimal hedging strategy for the Levy process. Finally in section 4 we will derive a natural generalization of the Black-Scholes option pricing formula for the case when the underlying security is modeled by a geometric TLP. This demonstrates that, unlike for the plain Levy process, the Black-Scholes framework is easily adapted to the truncated Levy paradigm.

\section{The Truncated Levy Distribution}

In this section we will outline the essential properties of the TLD and the TLF. Consider a general probability density function $(\mathrm{PDF}) P(x)$ and its characteristic function $(\mathrm{CF}) \hat{P}(k)$ defined by

$$
P(x)=\frac{1}{2 \pi} \int_{-\infty}^{\infty} d k \hat{P}(k) e^{i k x}, \quad \hat{P}(k)=\int_{-\infty}^{\infty} d x P(x) e^{-i k x}=\left\langle e^{-i k x}\right\rangle .
$$

Moments of the distribution can be found from the CF by using

$$
\left\langle x^{m}(t)\right\rangle=\left.\left(i^{m} \frac{\partial^{m}}{\partial k^{m}} \hat{P}(k, t)\right)\right|_{k=0} .
$$

Normalization of the PDF requires $\hat{P}(0)=1$. We will deal purely with symmetric $P(x)$ which in turn requires $\hat{P}(k)$ to be real and symmetric.

The symmetric Levy distribution is defined by the CF [12

$$
\hat{L}(k)=\exp \left(-c^{\alpha}|k|^{\alpha}\right), \quad 0<\alpha \leq 2
$$

where $c$ is the scale factor and $\alpha$ is the characteristic exponent. The full PDF for the Levy distribution is only known analytically when $\alpha=1$ (Cauchy distribution) and $\alpha=2$ (Gaussian distribution). However the value of the Levy distribution is known at the origin where

$$
L(x=0)=\frac{\Gamma(1 / \alpha)}{\pi \alpha c}
$$

and in the tails where (for $\alpha<2$ )

$$
L(x) \rightarrow \frac{c^{\alpha} \Gamma(1+\alpha) \sin \pi \alpha / 2}{\pi|x|^{\alpha+1}}, \quad x \rightarrow \infty .
$$

These 'fat' power law tails mean the fractional moments $\left\langle|x|^{\mu}\right\rangle$ are finite only for $\mu<\alpha$. In particular, for $\alpha<2$ the variance is infinite. 
The TLD is a generic description for a Levy distribution that has some cutoff far in the power law tails. Such a cutoff will ensure that the variance of the distribution is finite. One possible cutoff is the exponential function for which the CF has been shown to be [9]

$$
\hat{T}(k)=\exp \left[-\frac{c^{\alpha}}{\cos (\pi \alpha / 2)}\left(\left(k^{2}+\lambda^{2}\right)^{\alpha / 2} \cos \{\alpha \arctan (k / \lambda)\}-\lambda^{\alpha}\right)\right], \quad \alpha \neq 1 .
$$

With this CF the TLD in the tails takes the form

$$
T(x) \rightarrow \frac{c^{\alpha} \Gamma(1+\alpha) \sin (\pi \alpha / 2) e^{-\lambda|x|}}{\pi|x|^{1+\alpha}}, \quad|x| \rightarrow \infty .
$$

Clearly the TLD reduces to the Levy distribution when $\lambda=0$. The asymmetric generalization of (2.6) was also derived in [9]. However in the paper we will deal only with the symmetric version. Applying (2.2) to (2.6) we find the variance and kurtosis of the TLD to be

$$
\sigma^{2}=\frac{\alpha(1-\alpha)}{\cos (\pi \alpha / 2)} c^{\alpha} \lambda^{\alpha-2}, \quad k=\frac{\cos (\pi \alpha / 2)(\alpha-2)(\alpha-3)}{\alpha(1-\alpha) c^{\alpha} \lambda^{\alpha}}
$$

where the variance and kurtosis are defined by

$$
\sigma^{2}=\left\langle x^{2}\right\rangle, \quad k=\frac{\left\langle x^{4}\right\rangle}{\left\langle x^{2}\right\rangle^{2}}-3 .
$$

We can always set $c=1$ by scaling $x$ as $x \rightarrow \gamma x$. The CF of the scaled $x$ is again (2.6) but now with $c \rightarrow c \gamma$ and $\lambda \rightarrow \lambda / \gamma$. The kurtosis (2.8) and the exponent $\alpha$ will both remain fixed under scaling.

An important consequence for option pricing is that the exponential cutoff in (2.7) will ensure that exponential moments $\left\langle e^{n x}\right\rangle$ ( $n$ is any real number) exist for $n \leq \lambda$. The exponential moments can be found from the CF (2.6) simply by substituting $k^{2} \rightarrow-n^{2}$. This is equivalent to putting $k \rightarrow i n$ in (2.1) when the $\mathrm{CF}$ is symmetric and can be written as a function of $k^{2}$. After the substitution we can expand (2.6) in powers of $n^{2} / \lambda^{2}$ and find to first order that

$$
\left\langle e^{n x}\right\rangle \simeq \exp \left[\frac{n^{2}}{2}\left\langle x^{2}\right\rangle\right], \quad \lambda^{2} \gg n^{2}
$$

In the Gaussian case $(\lambda=0, \alpha=2)$ this is exact.

\subsection{Convergence to Gaussian}

Let us now consider $x$ to be the sum of $N$ independent and identically distributed random variables $x_{i}$ with a TLD defined by the CF (2.6). Following Mantegna and Stanley [8] we can refer to $x$ as a TLF. The CF of $x$ will be

$$
\hat{T}(k, N)=\exp \left[-\frac{c^{\alpha} N}{\cos (\pi \alpha / 2)}\left(\left(k^{2}+\lambda^{2}\right)^{\alpha / 2} \cos \{\alpha \arctan (k / \lambda)\}-\lambda^{\alpha}\right)\right], \quad \alpha \neq 1
$$


which is the same as (2.6) but with $c^{\alpha} \rightarrow N c^{\alpha}$. First consider the special case when each $x_{i}$ has a PDF described by a Levy distribution $(\lambda=0)$. The Levy distribution is also known as the Levy stable distribution. This is because the PDF of the rescaled variable $x N^{-1 / \alpha}$ is the same as that of $x_{i}$, that is the Levy distribution is stable under addition (the central limit theorem does not apply since the Levy distribution has infinite variance). We can also refer to this as the scaling or fractal property of the Levy distribution. On the other hand, for $\lambda>0$, the variance of $x_{i}$ is finite so the PDF of $x$ must approach a Gaussian by the central limit theorem. What is remarkable is that, as first pointed out by Mantegna and Stanley [8], the convergence of $x$ to a Gaussian occurs extremely slowly due to the stable property of the Levy distribution.

We can derive the crossover time $N_{c}$ for $x$ to converge to a Gaussian as follows. From (2.8) we know that the variance and kurtosis of $x$ are given by

$$
\sigma^{2}(N)=\frac{\alpha(1-\alpha)}{\cos (\pi \alpha / 2)} c^{\alpha} \lambda^{\alpha-2} N, \quad k(N)=\frac{\cos (\pi \alpha / 2)(\alpha-2)(\alpha-3)}{\alpha(1-\alpha) c^{\alpha} \lambda^{\alpha} N} .
$$

A useful qualitative model of the distribution of $x$ is that of a Gaussian in the central part out to the scale determined by the square root of the variance (2.12) (and therefore growing as $\sqrt{N}$ ). Beyond this scale we can think of the distribution being described by the tails $(2.7)$ (with $c^{\alpha} \rightarrow N c^{\alpha}$ ) which are slowing being consumed by the Gaussian part. We can then ask at what time $N_{c}$ does the scale set by the square root of the variance equal the cutoff scale $\lambda^{-1}$. We easily find the crossover time $N_{c}$ to be

$$
N_{c}=\frac{\cos (\pi \alpha / 2)}{\alpha(1-\alpha)} c^{-\alpha} \lambda^{-\alpha}
$$

A more rigorous method to derive this timescale is to find the time for the kurtosis of $x$ to decay away. From (2.12) we see that this timescale is consistent with (2.13) which justifies the simple qualitative picture of the convergence to a Gaussian. We will find this picture helpful later when option hedging is considered. Mantegna and Stanley 8 first derived the timescale $N_{c} \sim c^{-\alpha} \lambda^{-\alpha}$ using a method based on the probability of $x$ returning to the origin (this method gives a slightly different $\alpha$ dependent coefficient). This result can also be obtained from the Berry-Esseen theorem [13] and will be independent of the precise form of the cutoff. Clearly $N_{c}$ can be as large as one wishes by making $\lambda$ small enough.

\subsection{Parameter Fitting}

Parameter fitting to a TLF is no more complex than fitting data to a Levy distribution. The parameters $\alpha$ and $c$ can be obtained by fitting a Levy distribution to price increments (or log increments) on the smallest timescale such that linear correlations are negligible. A good rule of thumb is 30 minute increments. The timescale needs to be small so that we are operating well in the Levy regime of the TLF. Once $c$ and $\alpha$ are known we can extract the cutoff parameter $\lambda$ from the variance of the dataset using (2.12). This will fit the variance

of the data for a particular time $N$. From the point of view of option pricing fitting to the 
daily variance would seem the most appropriate choice. It would be appropriate to fit $\lambda$ using a large dataset to ensure a good description of the tails. Once $\alpha$ and $\lambda$ are determined we can expect these parameters to be relatively stable despite the well documented nonstationary behavior associated with a stochastic variance. These effects can be accounted for by a time dependent scale factor $c$. The non-stationary behavior is associated with variance measurements over small data sets (typically 1 month). This data will only sample the central part of the PDF which compared to $c$ is relatively insensitive to $\lambda$. The relative stability of the exponent $\alpha$ over time might be expected since it is invariant to scale changes. As a consequence sums of Levy distributed random variables with the same exponent $\alpha$, but different scale factor $c$, will still have a distribution described by the exponent $\alpha$. This stability has been confirmed in empirical studies on the S\&P 500 index [4].

There are several methods for fitting data to a Levy distribution (see Rachev and Mittnik [1] for a review). Here we adopt the method used by Mantegna and Stanley [4]. It is based on the relation

$$
T(x=0, N) \simeq L(x=0, N)=\frac{\Gamma(1 / \alpha)}{\pi \alpha c N^{1 / \alpha}}, \quad N \ll N_{c}
$$

which will be a good approximation in the Levy regime of the TLF $\left(N \ll N_{c}\right)$. In this method we can extract $c$ and $\alpha$ by fitting a power law decay to the probability of return to the origin for times $N \ll N_{c}$. Alternatively we can take the $\log$ of (2.14) and extract $c$ and $\alpha$ by a straight line fit. This is a very practical method because it is simple, it can be used on relatively small datasets (since $T(0, N)$ is the maximum of the distribution), and because of the easy availability of high frequency financial data. It is somewhat different to other methods in that it fits the peak of the distribution at various times rather than fitting the whole distribution at a single time.

We will demonstrate the parameter fitting process using high frequency equity data. We have used the All Ordinaries Index (AOI) which is the major Australian equity index. The dataset comprised the value of the AOI at 5 minute intervals from 1993-May/1997. From this, datasets describing the raw change in the AOI were constructed for 30 minutes, 1 hour, 2 hours, 3 hours and 1 day. The mean was then subtracted from these datasets. The time of 30 minutes was considered to be a good minimum time for which changes in the AOI could be considered to have negligible linear correlations. This is necessary in order to justify the use of (2.14). From these datasets the probability of zero change in the AOI was found and plotted against time in figure 1 (time is in units of 30 minutes with 1 trading day equal to 6 hours or $N=12$ ).

The data of figure 1 was very well fitted to a power law decay curve. From the curve of best fit and (2.14) it was found that $\alpha \simeq 1.2$ and $c \simeq 1.1$. For comparison in figure 1 the fit of $P(0)$ to a Gaussian process is shown where the Gaussian is fitted to the daily variance of 226 (as would occur in practice from an option pricing perspective) and extrapolated back to 30 minutes. In figure 2 the empirical PDF for the 30 minute data is shown along with the Levy PDF defined by the CF (2.11) with the parameters $\alpha=1.2, c=1.1, \lambda=0$ and $N=1$. We also show the Gaussian PDF which has been fitted to the variance of the daily data and extrapolated back to 30 minutes. The Levy PDF can be seen to be in good aggreement with 
the empirical PDF all the way out to to \pm 50 points which is approximately 12 standard deviations. The tails of the distribution are quite sensitive to the exponent $\alpha$ which we demonstrate by also showing the Levy PDF for $\alpha=1.4$. From this we can see that simple fitting method used has been quite effective. These results along with others [1, 4, 4] further support the LF/TLF model of financial data.

To fit $\lambda$ the dataset used was that of daily changes in the AOI between 1987-96. A large data set is required to fit $\lambda$ since this relates to the tails of the distribution. Substituting the daily variance of 345 and $N=12$ into (2.12), and using the same values for $\alpha$ and $c$ derived previously, we find that $\lambda=1 / 80$. With these parameter values we find that the Levy-Gaussian crossover time $(2.13)$ is $N_{c} \simeq 222$, or approximately 19 trading days. This is very close to 1 trading month (21 days) which previous studies have shown to be the timescale for which the Gaussian description becomes accurate [3], 田. Since $N_{c} \gg 1$ day the use of (2.14) out to 1 trading day is justified since this is well within the Levy regime of the TLF.

The daily dataset from 1987-96 is quite symmetric except in the far tails. The largest positive deviation in the dataset is 80 points. On the other hand there are several negative deviations beyond 100 points which all occured around the 1987 crash of -520 points. These facts suggest that an appropriate asymmetric model would be a symmetric Levy distribution with asymmetric cutoff parameters, rather than an asymmetric Levy distribution with symmetric cutoff parameter. The later model [9] would give asymmetry in the whole distribution rather than just in the far tails. In option pricing generally only part of the probability distribution is important. This suggests that a simple and effective approach to asymmetry would be to choose a cutoff parameter that best fits the part of the distribution that is relevant. To illustrate this we have derived the cutoff parameter $\lambda_{+}=1 / 41$ from the variance of positive deviations (203), and the cutoff parameter $\lambda_{-}=1 / 122$ derived from the variance of negative deviations (487). This latter cutoff parameter leads to a crossover time of approximately 30 days. We have used $\alpha=1.2, c=1.1$ and $N=12$ as before.

\section{Optimal Option Hedging}

In this section we will be concerned with the optimal hedging of call options for times to expiry which are less than the Levy-Gaussian crossover time. With 1 trading month as a typical crossover timescale we would be interested in times to expiry roughly less than 10 trading days. In this case the drift and probability of negative prices are negligible and we can consider the TLP as our model for the financial data.

Consider first the price $C\left(S_{0}, E, t\right)$ of a European call option at current time $t=0$, with exercise price $E$ due to expire in a time $t$. When the time to expiry is small the returns and interest rates can be neglected. The option price is then very well approximated by

$$
C\left(S_{0}, E, t\right) \simeq\langle\max (S-E, 0)\rangle=\int_{E}^{\infty} d S(S-E) P\left(S, t \mid S_{0}, 0\right)
$$

where $P\left(S, t \mid S_{0}, 0\right)$ is the driftless PDF of the underlying asset price $S(t)$. We know in 
advance that (3.1) will lead to the familiar volatility smile effect caused by the 'fat' tails of non-Gaussian PDF's like the TLD. These effects are well known and we will not consider them further. What is much less understood and discussed is the issue of option hedging for non-Gaussian models. It is to this that we now turn.

\subsection{Variance based hedging strategy}

A framework which can address the hedging issue is the Bouchaud-Sornette approach to option pricing and hedging [10, 11]. This approach starts by finding the variation in wealth for a call option seller. They find the wealth variation between times 0 and $t$ can be written as

$$
\left.\Delta W\right|_{0} ^{t}=C\left(S_{0}, E, t\right)-\max (S(t)-E, 0)+\int_{0}^{t} d \tau \phi\left(S_{\tau}\right) \dot{S}(\tau)
$$

where the first term is the option premium received at $t=0$, the second term describes the payoff at expiry $t$ and the third term describes the effect of trading where $\phi\left(S_{\tau}\right)\left(S_{\tau}=S(\tau)\right)$ is the amount of stock held. The interest rate is set at zero for clarity which will be a good approximation for short-medium term options. The option price is found by requiring $\left\langle\left.\Delta W\right|_{0} ^{t}\right\rangle=0$. This leads to

$$
C\left(S_{0}, E, t\right)=\langle\max (S(t)-E, 0)\rangle-\int_{0}^{t} d \tau\left\langle\phi\left(S_{\tau}\right)\right\rangle\langle\dot{S}(\tau)\rangle
$$

where the increment $\dot{S}(\tau)$ is in the future and assumed to be independent of $S(\tau)$ (this assumption can be relaxed [10]). What is remarkable about the Black-Scholes case (Gaussian or log-Gaussian models) is that there exists a trading strategy $\phi^{*}\left(S_{\tau}\right)$ such that $\left.\Delta W\right|_{0} ^{t}$ can be made to vanish. In this case the rate of return dependence in (3.3) cancels and the BlackScholes result follows [10]. For more general pricing models a riskless hedge does not exist and in these cases the Black-Scholes framework fails. However the Bouchaud-Sornette method is easily adapted to these more realistic situations. Although in general a riskless hedge does not exist, it is possible to find an optimal trading strategy $\phi^{*}\left(S_{\tau}\right)$ that will minimize some appropriate measure of risk. An obvious choice for a risk measure is the variance of the wealth distribution $\left\langle\left.\Delta W^{2}\right|_{0} ^{t}[\phi]\right\rangle$, or its 4 th moment which would place more weight on the tails. The optimal trading strategy can be easily computed for any PDF describing the price $S(t)$. In these more general cases the option price (3.3) (evaluated at $\phi^{*}$ ) will depend on the rate of return. However the return can be safely set to zero for options less than a few months to expiry (see Aurell et-al []]). In this case the second term on the right hand side of (3.3) will vanish and (3.1) will be a good approximation. However the option price (3.3) will need to be corrected by a risk premium whose scale will be set by the residual risk $\sqrt{\left\langle\left.\Delta W^{2}\right|_{0} ^{t}\left[\phi^{*}\right]\right\rangle}$. This risk premium can account for the bid-ask spreads in option prices.

Consider the special case of a pricing model in which the price increments are uncorrelated, stationary and have zero mean (these assumptions can be relaxed). This includes the TLP and will be a good model for options roughly less than a month to expiry. In this case 
the hedging strategy that minimizes the variance of the wealth distribution has been shown to be 10, 11]

$$
\phi^{*}\left(S_{0}, E, t\right)=\frac{1}{\sigma^{2} t} \int_{E}^{\infty} d S(S-E)\left(S-S_{0}\right) P\left(S-S_{0}, t\right)
$$

where $P\left(S-S_{0}, t\right)$ is the conditional PDF of the pricing model with initial price $S_{0}$ and $\sigma^{2} t$ is the variance of the model. The optimal hedge $\phi^{*}$ ranges between 0 and 1 since we are dealing with an option on one unit of the underlying security. For at-the-money options $\left(E=S_{0}\right)$ we easily find from (3.4) that $\phi^{*}=1 / 2$ for any pricing model. For options out-of-the-money $\left(E>S_{0}\right)$ we have $\phi^{*}<1 / 2$ and for options in-the-money $\left(E<S_{0}\right)$ we have $\phi^{*}>1 / 2$.

For the special case of the Gaussian pricing model with PDF

$$
G\left(S-S_{0}, t\right)=\frac{1}{\sqrt{2 \pi \sigma^{2} t}} \exp \left(-\frac{\left(S-S_{0}\right)^{2}}{2 \sigma^{2} t}\right)
$$

the optimal hedging strategy (3.4) reduces to the Gaussian Black-Scholes result

$$
\phi^{*}\left(S_{0}, E, t\right)=\frac{\partial}{\partial S_{0}} \int_{E}^{\infty} d S(S-E) G\left(S-S_{0}, t\right)=N\left(\frac{S_{0}-E}{\sigma \sqrt{t}}\right)
$$

where $N$ is defined in (4.17). This result can also be shown to be the probability of exercise of the option. This hedging strategy is clearly just the derivative of the option price with respect to $S_{0}$. This is the well known delta hedging result and can be expressed more generally as

$$
\Delta\left(S_{0}, E, t\right)=\frac{\partial}{\partial S_{0}} C\left(S_{0}, E, t\right) \simeq \frac{\partial}{\partial S_{0}} \int_{E}^{\infty} d S(S-E) P\left(S-S_{0}, t\right)
$$

where we have used the approximate option price (3.1). This is a riskless hedging strategy only for Gaussian and log-Gaussian models. However it can be used to give the hedge value for other pricing models.

Consider the PDF for the TLP which has the tail form

$$
T\left(S-S_{0}, t\right) \simeq \frac{c^{\alpha} t \Gamma(1+\alpha) \sin \pi \alpha / 2}{\pi\left|S-S_{0}\right|^{1+\alpha}} \exp \left(-\lambda\left|S-S_{0}\right|\right), \quad \frac{\left|S-S_{0}\right|}{\sqrt{\sigma^{2} t}}>1 .
$$

The scale where this approximation becomes valid is set by the Levy process diffusion scale $c t^{1 / \alpha}$. The variance of the TLP will always be greater than this scale for times less than the Levy-Gaussian crossover time. We will always be interested in times less than this which makes the condition on $\left|S-S_{0}\right|$ appropriate. We wish to compare for this model, the optimal Bouchaud-Sornette hedging strategy (3.4) with the delta hedge (3.7). We will also compare the TLP hedges with the Gaussian delta hedge (3.6). Applying the PDF (3.8) to (3.4) we find that the optimal Bouchaud-Sornette hedge becomes

$$
\phi^{*}\left(S_{0}, E, t\right) \simeq \frac{\Gamma(1+\alpha) \sin \pi \alpha}{2 \pi \alpha(\alpha-1)^{2}}\left[y^{2-\alpha} e^{-y}+(1-y-\alpha) \Gamma(2-\alpha, y)\right], \quad \frac{E-S_{0}}{\sqrt{\sigma^{2} t}}>1,
$$


where

$$
y=\left(\frac{t}{t_{c}}\right)^{1 / 2} \frac{\left|E-S_{0}\right|}{\sqrt{\sigma^{2} t}}=\lambda\left|E-S_{0}\right|
$$

and

$$
\Gamma(a, x)=\int_{x}^{\infty} d t e^{-t} t^{a-1}
$$

is the incomplete gamma function. In $(3.10), t_{c}$ is the same as the discrete time LevyGaussian crossover time defined in (2.13). For $S_{0}>E$ we have

$$
\phi^{*}\left(S_{0}, E, t\right) \simeq 1-\frac{\Gamma(1+\alpha) \sin \pi \alpha}{2 \pi \alpha(\alpha-1)^{2}}\left[y^{2-\alpha} e^{-y}+(1-y-\alpha) \Gamma(2-\alpha, y)\right], \quad \frac{S_{0}-E}{\sqrt{\sigma^{2} t}}>1
$$

Consider next the delta hedging strategy based on the TLP. Applying the PDF (3.8) to (3.7) we find

$$
\Delta\left(S_{0}, E, t\right) \simeq \frac{t}{t_{c}} \frac{\Gamma(1+\alpha) \sin \pi \alpha}{2 \pi \alpha^{2}(\alpha-1)^{2}}\left[y^{1-\alpha} e^{-y}-(\alpha-1) y^{-\alpha} e^{-y}-\Gamma(2-\alpha, y)\right], \quad \frac{E-S_{0}}{\sqrt{\sigma^{2} t}}>1
$$

and for $S_{0}>E$

$\Delta\left(S_{0}, E, t\right) \simeq 1-\frac{t}{t_{c}} \frac{\Gamma(1+\alpha) \sin \pi \alpha}{2 \pi \alpha^{2}(\alpha-1)^{2}}\left[y^{1-\alpha} e^{-y}-(\alpha-1) y^{-\alpha} e^{-y}-\Gamma(2-\alpha, y)\right], \quad \frac{S_{0}-E}{\sqrt{\sigma^{2} t}}>1$.

The Gaussian delta hedge (3.6) is exactly equal to the probability of exercise of the option for the Gaussian pricing model (3.5). The TLP delta hedge (3.13-14) is also equal to the probability of exercise when calculated with the tail approximation (3.8).

In figures 3 and 4 we plot and compare the TLP Bouchaud-Sornette optimal hedge (3.9), the TLP delta hedge (3.13) and the Gaussian delta hedge (3.6). We are using $E>S_{0}$ which means the options are out-of-the-money and have a low hedge value and probability of exercise. We plot the hedge value against the ratio of $E-S_{0}$ and the standard deviation of the pricing model. No parameter values need to be specified in the Gaussian delta hedge (3.6). For the hedges (3.9) and (3.13), we know from (3.10) that we need to specify the exponent $\alpha$ and the ratio of the time to expiry $t$ and the Levy-Gaussian crossover time $t_{c}$. In section 2 we obtained $\alpha=1.2$ and a Levy-Gaussian crossover time of approximately 20 trading days. We have used these parameters with the expiry times of 1 and 5 days in figures 3 and 4 respectively. In figure 3 we see that Bouchaud-Sornette optimal hedge leads to hedge values that are, in percentage terms, significantly greater than those obtained with both delta hedges. The relative difference increases as $E-S_{0}$ grows. In figure 5 the differences are not as great, but in percentage terms they are still very large.

\subsection{Distribution based hedging strategy}

The Bouchaud-Sornette method will fail for models such as the Levy process which have infinite moments. Although the TLP has finite variance, in the early Levy dominated regime 
a distribution based method to find an optimal trading strategy may be preferable to a moment based method. Below we will adapt to a truncated Levy model a simple method used by Bouchaud et-al [2, 11] to find the optimal hedging strategy for Levy models.

Consider the case where $\phi$ is the final hedge value some time $t$ from expiry. Then from (3.2) we find that the change in wealth over this period is (ignoring the premium)

$$
\Delta W=I\left((\phi-1)\left(S_{t}-S_{0}\right)+E-S_{0}\right)+(1-I) \phi\left(S_{t}-S_{0}\right), \quad 0 \leq \phi \leq 1
$$

where $I=1$ for $S_{t}>E$ and $I=0$ for $S_{t}<E$. Now we make the key assumption that $I$ can be treated as an independent random variable that takes on values 1 and 0 with probabilities $\mathcal{P}$ and $1-\mathcal{P}$, where $\mathcal{P}$ is the probability the option will be exercised. Note that both $\phi$ and $\mathcal{P}$ are functions of $\left(S_{0}, E, t\right)$. We can write the change in wealth $\left.\Delta W\right|_{S_{t}-S_{0}}$, due only to the change in price as

$$
\left.\Delta W\right|_{S_{t}-S_{0}}=I(1-\phi)\left(S_{0}-S_{t}\right)+(1-I) \phi\left(S_{t}-S_{0}\right)
$$

In most realizations of $I$ and $S_{t}-S_{0}$, this will lead to a loss which we denote by $l$. We assume that $S_{t}-S_{0}$ is described by a TLP which has the PDF (3.8) in the tails. Due to the independence assumption of $I$ we can easily write down the tail PDF of the loss $l$ using (3.8). From this we find that the probability of a loss greater than $l_{*}$ is

$$
\mathcal{P}\left(l>l_{*}\right) \sim(1-\mathcal{P}) \phi^{\alpha} \int_{l_{*}}^{\infty} d l \frac{e^{-\lambda_{-} l / \phi}}{l^{1+\alpha}}+\mathcal{P}(1-\phi)^{\alpha} \int_{l_{*}}^{\infty} d l \frac{e^{-\lambda_{+} l /(1-\phi)}}{l^{1+\alpha}}
$$

We can write $l_{*}=1 /\left(2 \lambda_{*}\right)$ which is the average loss suffered to an event $\pm 1 / \lambda_{*}$. Thus we can consider minimizing losses above $l_{*}$ to be equivalent to minimizing losses to events beyond $\pm 1 / \lambda_{*}$. So after performing the integrals we can write (3.17) as

$$
\mathcal{P}\left(l>l_{*}\right) \sim\left(1-\mathcal{P}\left(S_{0}, t\right)\right) \lambda_{-}^{\alpha} \Gamma\left(-\alpha, \beta_{-}\right)+\mathcal{P}\left(S_{0}, t\right) \lambda_{+}^{\alpha} \Gamma\left(-\alpha, \beta_{+}\right)
$$

where

$$
\beta_{-}=\frac{\lambda_{-}}{2 \lambda^{*} \phi}, \quad \beta_{+}=\frac{\lambda_{+}}{2 \lambda^{*}(1-\phi)} .
$$

We wish to find the optimal trading strategy $\phi^{*}$ that will minimize the probability (3.18). This is obtained by by taking the derivative of (3.18) with respect to $\phi$, setting the left hand side to zero to obtain

$$
0=(1-\mathcal{P}) \phi^{\alpha-1} \exp \left(-\beta_{-}\right)-\mathcal{P}(1-\phi)^{\alpha-1} \exp \left(-\beta_{+}\right)
$$

and solving this equation for $\phi^{*}$ as a function of $\mathcal{P}$. For $\lambda=0,(3.20)$ becomes independent of $\lambda_{*}$ and it can be solved to obtain the Levy optimal hedge

$$
\phi^{*}\left(S_{0}, E, t\right)=\frac{\mathcal{P}^{\xi}\left(S_{0}, E, t\right)}{\mathcal{P}^{\xi}\left(S_{0}, E, t\right)+\left(1-\mathcal{P}\left(S_{0}, E, t\right)\right)^{\xi}}, \quad \xi=1 /(\alpha-1)
$$


first derived by Bouchaud et-al [2, [1]. For $\alpha=2$ the Gaussian delta hedge $\phi^{*}=\mathcal{P}$ is recovered.

The Levy hedge (3.21) is of fundamental interest as it shows that a hedging strategy can be derived even for a model with infinite variance. However what is required in practice is a trading strategy that interpolates between the Levy hedge and the Gaussian hedge over the crossover timescale (2.13). This follows from the central limit theorem which ensures that the optimal hedge tends to the Gaussian hedge (this is not the case for the geometric TLP discussed in section 4). Clearly we want to choose $\lambda_{*}^{-1}$ to be the smallest possible value such that the tail form of the PDF will hold. The scale beyond which this holds is set by the Levy diffusion scale $c t^{1 / \alpha}$. A good choice is $\lambda_{*}^{-1}=\sigma \sqrt{t}$ which is the standard deviation of $S_{t}-S_{0}$. This scale will always be greater than the Levy diffusion scale for times less than the crossover timescale. This is fine since this method of hedging will only be appropriate for these times. The choice for $\lambda_{*}$ is also consistent with the qualitative picture, discussed in section 2.1, for the convergence of the TLP to a Gaussian.

In figure 5 we plot the optimal hedging strategy against the probability of exercise obtained by a numerical solution of (3.20). We have chosen as parameters those obtained in section $2.2(\alpha=1.2$ and $\lambda=1 / 80)$ which gave a Levy-Gaussian crossover time of approximately 19 trading days. Using $\lambda_{*}^{-1}=\sigma \sqrt{t}$, we plot the optimal hedge at 2 days $\left(\lambda / \lambda_{*} \simeq 0.33\right), 10$ days $\left(\lambda / \lambda_{*} \simeq 0.73\right)$ and 20 days $\left(\lambda / \lambda_{*} \simeq 1.03\right)$ along with the Gaussian hedge $(\alpha=2, \lambda=0)$ and the Levy hedge $(\lambda=0)$. The hedging strategies have the very nice feature of a well defined evolution from the Levy hedge (3.21) to the Gaussian hedge in about 10 days. Between 10 and 20 days there is a much slower creep away from the Gaussian hedge. From this we can tentatively conclude that the hedging strategy defined by (3.20) could be reliably used out to time $t_{*}$ defined by $\lambda_{*} \simeq \lambda / 0.73$, or approximately $1 / 2$ the crossover time (2.13). Beyond this the Bouchaud-Sornette variance based method would be appropriate. In figure 5 we see that for options with a small probability of exericise, the difference between the optimal hedge and the Gaussian Black-Scholes hedge is large in percentage terms. This is consistent with what was found in section 3.1.

Figure 6 is similar to figure 5 except that we have used the asymmetric cutoff parameters $\lambda_{+}=1 / 41$ and $\lambda_{-}=1 / 122$ discussed in section 2.2. The symmetric cutoff parameter $\lambda=1 / 80$ was used in the variance required to describe the growth in $\lambda_{*}$. This case gives an optimal hedge that involves holding less stock than in the symmetric case. This is what we expect since the asymmetric case has more weight in the negative tails and negative price changes favor holding zero stock. Plots of the ratio of the asymmetric/symmetric hedging strategies of figures 5 and 6 approximately range between 0.7 and 1 for exercise probabilities between 0 and 1 respectively.

\section{Option Pricing}

Here we will derive a generalization of the Black-Scholes option pricing formula for the case where the underlying security is modeled by a geometric TLP. We will see that the BlackScholes framework is easily adapted to the truncated Levy paradigm, a feature not shared 
by the plain Levy or geometric Levy process.

The Black-Scholes option pricing theory [14 is based on the geometric Brownian Motion (GBM) paradigm of financial market dynamics [15]. A natural generalization of GBM is to define the geometric TLP $S(t)$ by

$$
S(t)=S_{0} \exp \left[x(t)+\mu t-\frac{1}{2} \sigma^{2} t\right]
$$

where $x(t)$ is a stochastic process defined by the CF

$$
\hat{T}_{g}(k, t)=\exp \left[-\frac{c_{x}^{\alpha} t}{\cos (\pi \alpha / 2)}\left(\left(k^{2}+\lambda_{x}^{2}\right)^{\alpha / 2} \cos \left\{\alpha \arctan \left(k / \lambda_{x}\right)\right\}-\lambda_{x}^{\alpha}\right)\right], \quad \alpha \neq 1,
$$

$\sigma^{2} t$ is the variance of $x(t)\left(\sigma^{2}\right.$ is defined in (2.8)) and $\mu$ will be shown to be the rate of return. A time dependent variance can be considered by replacing $\sigma^{2} t$ with $\int_{0}^{t} d s \sigma^{2}(s) d s$. This time dependence will derive from a time dependent scale factor $c_{x}(t)$ in (4.2) with $\alpha$ and $\lambda_{x}$ kept constant. GBM is recovered by setting $\alpha=2$ and $\lambda_{x}=0$ in the CF (4.2). Using (2.8) this gives $2 c_{x}^{2}=\sigma^{2}$.

Using (2.10) we find the moments of the geometric TLP are

$$
\left\langle S^{n}(t)\right\rangle \simeq S_{0}^{n} \exp \left[n \mu t+n(n-1) \sigma^{2} t / 2\right], \quad \lambda_{x}^{2} \gg n^{2} .
$$

From this we see that $\mu$ is the rate of return. This expression is an approximation to first order in $n^{2} / \lambda_{x}^{2}$ and is exact for GBM. The approximation breaks down for very high order moments which is direct consequence of the absence of a central limit theorem for multiplicative processes like (4.1). But how do we know that the condition $\lambda_{x}^{2} \gg n^{2}$ will hold? Consider short timescales where $x(t)$ is small and we can write (4.1) as

$$
S(t) \simeq S_{0}+S_{0} x(t)
$$

Under this approximation $S(t)$ reduces to the plain TLP. Equation (4.4) will be a valid approximation when $\sqrt{\sigma^{2} t} \ll 1$. This will apply for time periods typically of the order of 1 month or less. Fitting data to raw changes in the price $S(t)$, as in section 2.2 , effectively fits $S_{0} x(t)$ to a TLP which is described by the parameters $\alpha, c$ and $\lambda$. We can then extract the parameters $c_{x}, \lambda_{x}$ of the CF of $x(t)$, by the relations $c_{x}=c / S_{0}$ and $\lambda_{x}=S_{0} \lambda$ ( $\alpha$ is invariant under scaling). We know that $\lambda_{x}$ must obey $\lambda_{x} \gg 1$ because the original cutoff scale $\lambda^{-1}$ of the TLP must be much smaller than $S_{0}$ to ensure the price stays positive. We have found that fitting daily data to change in log price leads to $\lambda_{x}$ typically in the range of 20-30.

Lets now consider the price $C\left(S_{0}, E, t\right)$ of a European call option at time $t=0$ with exercise price $E$ due to expire in time $t$. We will assume that the price of the underlying asset $S(t)$ follows a geometric TLP (4.1) with $S_{0}$ the current price. A simple but ad hoc approach to option pricing is to apply the the risk-neutral approach 15 in exactly the same way it is used with the GBM model. In this case the option price if given by

$$
C\left(S_{0}, t\right)=e^{-r t}\langle\max (S-E, 0)\rangle=e^{-r t} \int_{E}^{\infty} d S(S-E) T_{g}\left(S, t \mid S_{0}, 0\right)
$$


where $T_{g}\left(S, t \mid S_{0}, 0\right)$ is the PDF of the geometric TLP with $\mu$ set equal to $r$. Using $\int_{E}^{\infty} \rightarrow$ $\int_{0}^{\infty}-\int_{0}^{E}$, the normalization of $T_{g}\left(S, t \mid S_{0}, 0\right)$ and (4.3) we find that

$$
C\left(S_{0}, t\right) \simeq S_{0}-E e^{-r t}-e^{-r t} \int_{0}^{E} d S(S-E) T_{g}\left(S, t \mid S_{0}, 0\right), \quad \lambda_{x}^{2} \gg 1 .
$$

We can write the PDF of $S(t)$ in the form

$$
T_{g}\left(S, t \mid S_{0}, 0\right) d S=\frac{d x}{\pi} \int_{0}^{\infty} d k \hat{T}_{g}(k, t) \cos k x
$$

where $\hat{T}_{g}(k, t)$ is the CF of $x(t)$ defined by (4.2), and from (4.1) we have (with the risk-neutral measure)

$$
x=\ln \left(S / S_{0}\right)-r t+\frac{1}{2} \sigma^{2} t .
$$

We can substitute (4.7) into (4.6) and change the order of integration. Using the integrals

$$
\int_{-\infty}^{x_{e}} d x \cos k x=\frac{\sin k x_{e}}{k}+\pi \delta(k)
$$

and

$$
\int_{-\infty}^{x_{e}} d x e^{x} \cos k x=e^{x_{e}} \frac{\left(\cos k x_{e}+k \sin k x_{e}\right)}{1+k^{2}}
$$

we find the call option price is given by

$$
C\left(S_{0}, E, t\right) \simeq S_{0}-\frac{1}{2} E e^{-r t}+\frac{E e^{-r t}}{\pi} \int_{0}^{\infty} d k \hat{T}_{g}(k, t)\left(\frac{\sin k x_{e}-k \cos k x_{e}}{k\left(1+k^{2}\right)}\right), \quad \lambda_{x}^{2} \gg 1
$$

with $x_{e}$ defined by

$$
x_{e}=\ln \left(E / S_{0}\right)-r t+\frac{1}{2} \sigma^{2} t .
$$

We found $\lambda_{x}$ to be in the range 20-30. This means the approximation (4.11) will be very good.

The Black-Scholes result is recovered from (4.11) by writing

$$
\hat{T}_{g}(k, t)=\exp \left(-\sigma^{2} t k^{2} / 2\right)
$$

and using the identities

$$
\begin{gathered}
\frac{\sin k x_{e}-k \cos k x_{e}}{k\left(1+k^{2}\right)}=\frac{\sin k x_{e}}{k}-\frac{\left(k \sin k x_{e}+\cos k x_{e}\right)}{1+k^{2}} \\
\int_{0}^{\infty} d k \frac{e^{-p k^{2}}}{\gamma^{2}+k^{2}}(k \sin k x+\gamma \cos k x)=\pi e^{p \gamma^{2}-x \gamma} N\left(\frac{x-2 \gamma p}{\sqrt{2 p}}\right), \quad \gamma>0
\end{gathered}
$$


and

$$
\int_{0}^{\infty} d k e^{-p k^{2}} \frac{\sin k x}{k}=\frac{\pi}{2}-\pi N\left(\frac{-x}{\sqrt{2 p}}\right)
$$

where

$$
N(x)=\frac{1}{\sqrt{2 \pi}} \int_{-\infty}^{x} d y e^{-y^{2} / 2}, \quad N(x)=1-N(-x) .
$$

We then obtain the Black-Scholes option pricing formula [14]

$$
C\left(S_{0}, E, t\right)=S_{0} N(d)-E e^{-r t} N\left(d-\sqrt{\sigma^{2} t}\right)
$$

where

$$
d=\frac{\sigma^{2} t-x_{e}}{\sqrt{\sigma^{2} t}}
$$

Its important to emphasize that the option price (4.11) will not approach the Black-Scholes result (4.18) as the time to expiry increases. This is because the central limit theorem does not apply to multiplicative processes like the geometric TLP [18].

\section{Conclusion}

In this paper we have further demonstrated that the TLP can effectively model the empirical successes of both the Levy and Gaussian distributions at short and long timescales respectively. In option theory a major disincentive for using non-Gaussian based models is the absence of a riskless hedge. This makes it impossible to apply the Black-Scholes option pricing framework in anything other than an ad hoc way. We therefore discussed how the Bouchaud-Sornette approach to option theory provides a relatively simple solution to the problem of option pricing and optimal hedging in non-Gaussian models. We applied this optimal hedging strategy to the TLP pricing model and compared it with the delta hedging strategy for both the TLP and Gaussian models. Significant differences were found. We then discussed an alternative and computationally simple tail distribution based hedging strategy appropriate for the Levy regime of the TLP. For a TLP with exponential cutoff, the exponential moments exist and this allowed us to derive in section 4 a natural generalization of the Black-Scholes option pricing formula for the case of a geometric TLP pricing model. This is not possible for the geometric Levy process, an important reason for the lack of interest in this model.

Further theoretical work could involve deriving CF's of TLD's with alternative cutoffs such as stretched exponential tails, or models with asymmetric cutoff parameters. Stretched exponential tails are interesting technically because the Laplace transform of the distribution would then exist [16]. An asymmetric cutoff will give a PDF that is symmetric in the center but asymmetric in the far tails. This was how asymmetry occurred in the daily data set of the AOI considered in this paper. Also of great practical importance is the construction of efficient algorithms for the numerical simulation of random variables with a TLD. It may be possible to adapt existing methods for the simulation of Levy distributed random variables 
[17. With simulations we could test the effectiveness of the tail based hedging strategy against the variance based method. This is important since uncontrolled approximations had to be made in deriving the tail based strategy. The simple TLP model studied here does not address the apparent stochastic dynamics of the volatility along with its long range power law correlation function [6]. A challenge for the future is to construct pragmatic models which can describe these properties as well as the those of the distribution considered here.

Acknowledgement: I would like to thank the Australia Research Council for their generous support of this research through an Australian Postdoctoral Research Fellowship.

\section{References}

[1] B.B. Mandelbrot, Journal of Business 36, 394 (1963); B.B. Mandelbrot, Journal of Business 40, 394 (1967); E. Fama, Management Science 11, 404 (1965); J. H. McCulloch, Journal of Business 51, No 4, (1978); R.N. Mantegna, Physica A 179, 232 (1991); S.T. Rachev and S. Mittnik, Econometric Reviews 12, 261 (1993); E.E. Peters, Fractal Market Analysis, John Wiley and Sons, 1994.

[2] D. Edelman, Abacus 31, 113 (1995); J. P. Bouchaud, D. Sornette and M. Potters in Proceedings of the Newton Institute session on Mathematical Finance eds. M. Dempster and S. Pliska (Cambridge University Press, 1997).

[3] V. Akgiray and G.G. Booth, J. Business Econ. Statist. 6, 51 (1988).

[4] R.N. Mantegna and H.E. Stanley, Nature 376, 46 (1995); R.N. Mantegna and H.E. Stanley, Physica A 239, 225 (1997); R.N. Mantegna in The Physics of Complex Systems, Proceedings of the International School of Physics Enrico Fermi, Vol 134, eds. F. Mallamace and H.E. Stanley (IOS Press, Amsterdam 1997).

[5] D.G. Hobson, A Review of Stochastic Volatility Models (1996), available at: http://www.maths.bath.ac.uk/ Oz/papers.htm

[6] R. Cont, preprint cond-mat/9705075; Y. Liu et-al, preprint cond-mat/9706021; P. Cizeau et-al, preprint cond-mat/9708143; A. Arneodo et-al, preprint condmat/9708012; B. Holdom, preprint cond-mat/9709141.

All available at: http://xxx.lanl.gov

[7] R.N. Mantegna and H.E. Stanley, Nature 383, 587 (1996); A. Arneodo et al, preprint cond-mat/9607120 at http://xxx.lanl.gov; R. Cont et al, preprint cond-mat/9705087 at http://xxx.lanl.gov; J. P. Bouchaud, D. Sornette and M. Potters in Proceedings of the Newton Institute session on Mathematical Finance eds. M. Dempster and S. Pliska (Cambridge University Press, 1997); E. Aurell, J.P. Bouchaud, M. Potters and K. Zyczkowski, European Financial Management, (in press 1997). 
[8] R.N. Mantegna and H.E. Stanley, Phys. Rev. Lett 73, 2946 (1994); R.N. Mantegna and H.E. Stanley in Levy Flights and Related Topic in Physics eds. M.F. Shlesinger, G.M. Zaslavsky and U. Frisch (Springer, Berlin, 1995).

[9] I. Koponen, Phys. Rev. E 52, 1197 (1995).

[10] J.P. Bouchaud and D. Sornette, J. Phys. I France 4, 863 (1994); J.P. Bouchaud and D. Sornette, J. Phys. I France 5, 219 (1995).

[11] J.P. Bouchaud, G. Iori and D. Sornette, Risk 9(3), 61 (1996).

[12] V. Zolotarev, One-Dimensional Stable Distributions, American Mathematical Society, Providence RI (1986); J.P. Bouchaud and A. Georges, Physics Reports 195, 125 (1990).

[13] M.F. Shlesinger, Phys. Rev. Lett 74, 4959 (1995).

[14] F. Black and M. Scholes, Journal of Political Economy 81, 635 (1973).

[15] see for eg. J. Hull, Options, Futures, and other Derivatives 3rd ed., Prentice Hall, NJ (1997).

[16] D. Sornette, J. Phys. I France 7, 1155-1171 (1997).

[17] J.M. Chambers, C.L. Mallows and B. Stuck, J. Amer. Statist. Assoc. 71, 340 (1976); R. Weron, Statist. Probab. Lett 28, 165 (1996); R.N. Mantegna, Phys. Rev. E 49, 4677 (1994).

[18] S. Redner, Am. J. Phys. 58, 267 (1990); J.M. Deutsch, Physica A 208, 433 (1994). 


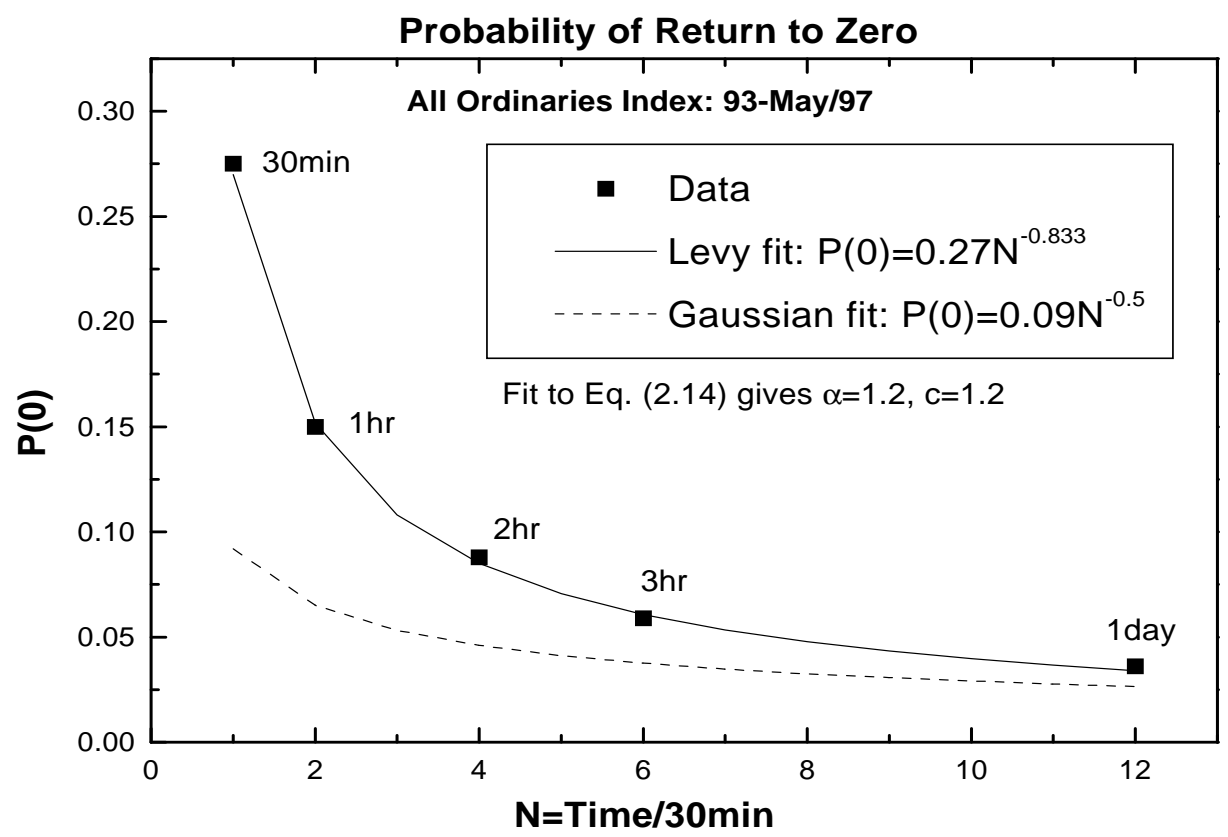

Figure 1: Fit of Eq. (2.14) to high frequency data

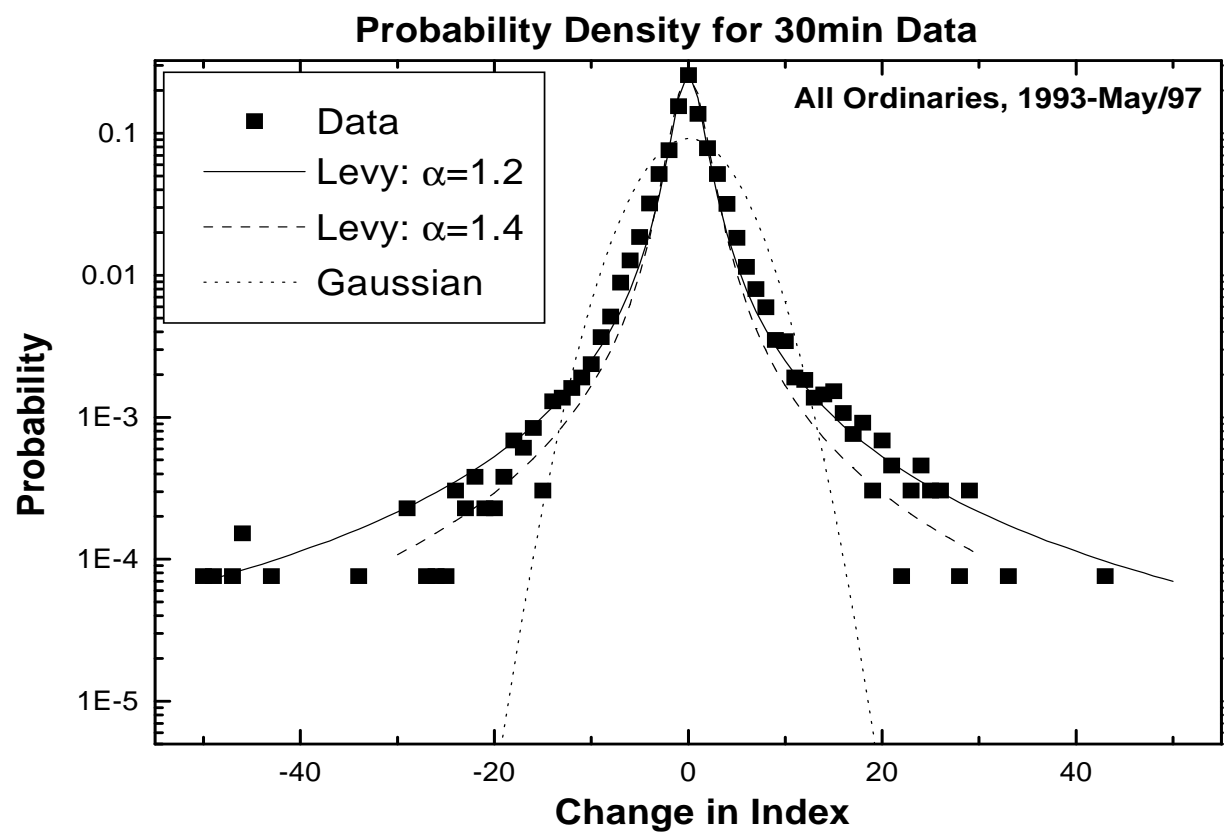

Figure 2: Fit of the Levy PDF to 30 minute data using parameters derived from figure 1 


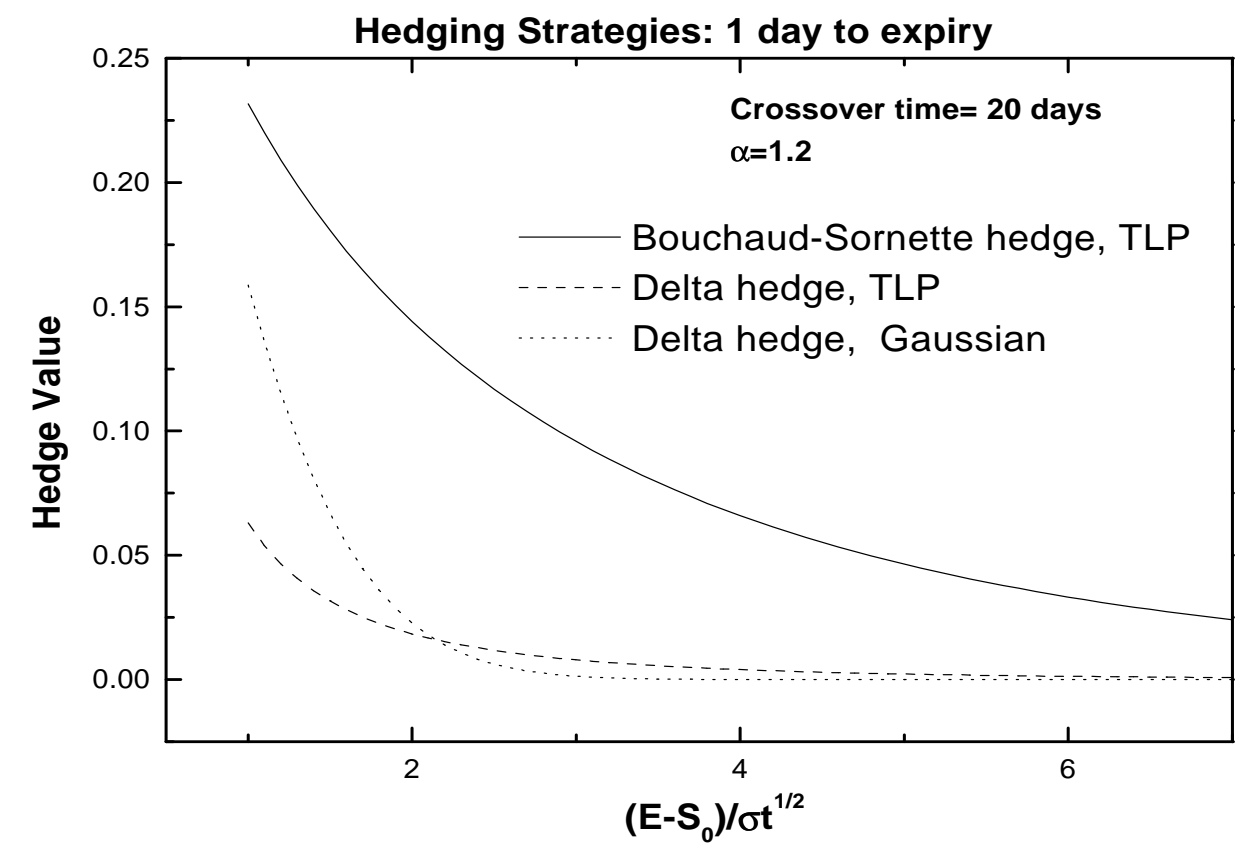

Figure 3: Plot of the the TLP Bouchaud-Sornette optimal hedge (3.9), the TLP delta hedge (3.13) and the Gaussian delta hedge (3.6).

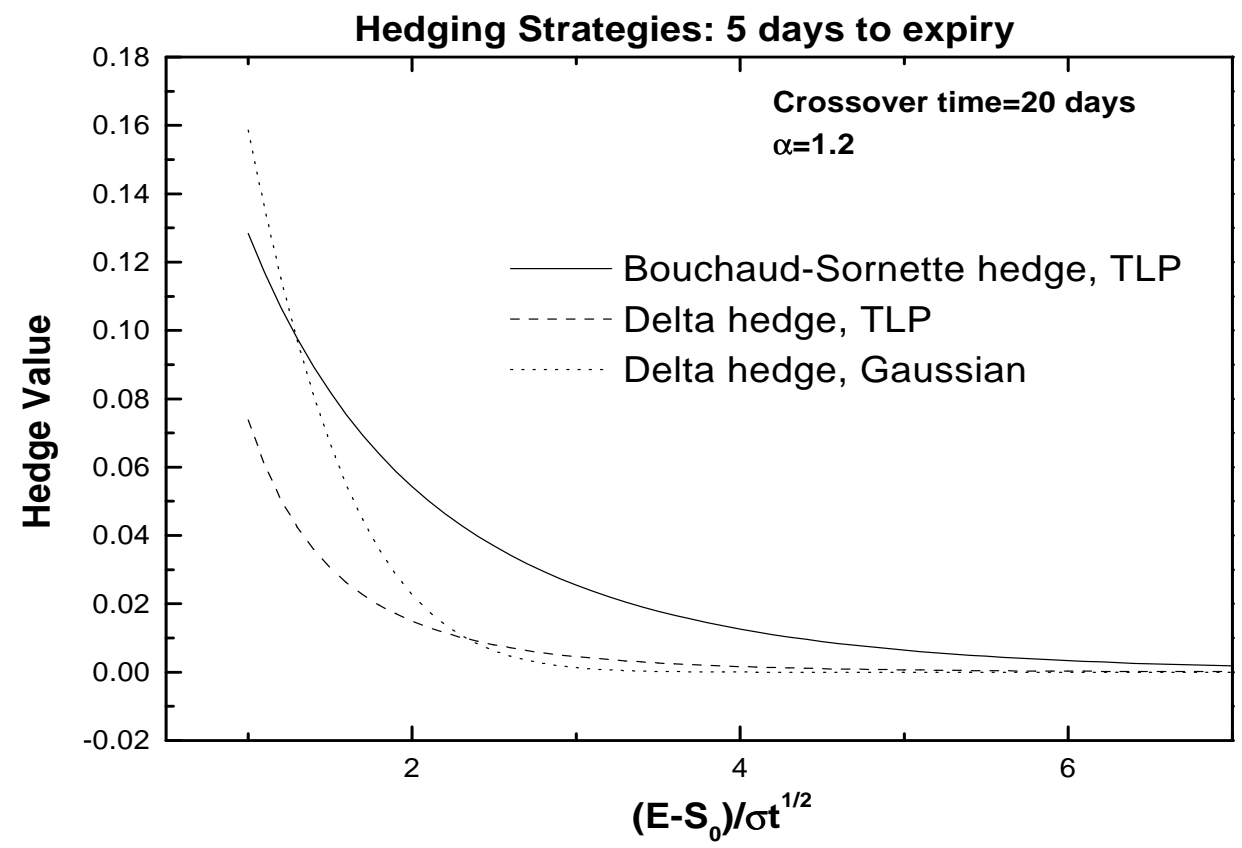

Figure 4: Same as figure 3 except 5 days to expiry 


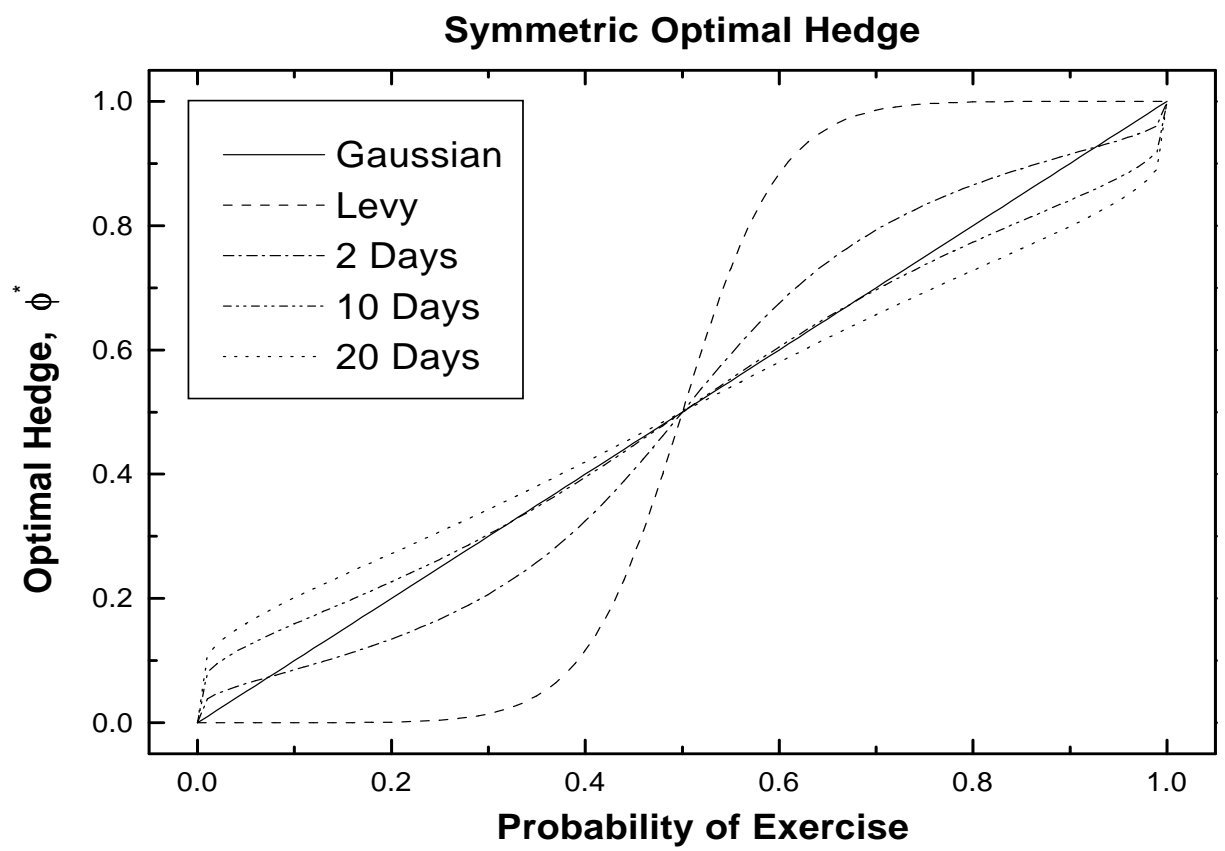

Figure 5: Solution to Eq. (3.20) with $\alpha=1.2$ and $\lambda_{-}=\lambda_{+}=1 / 80$.

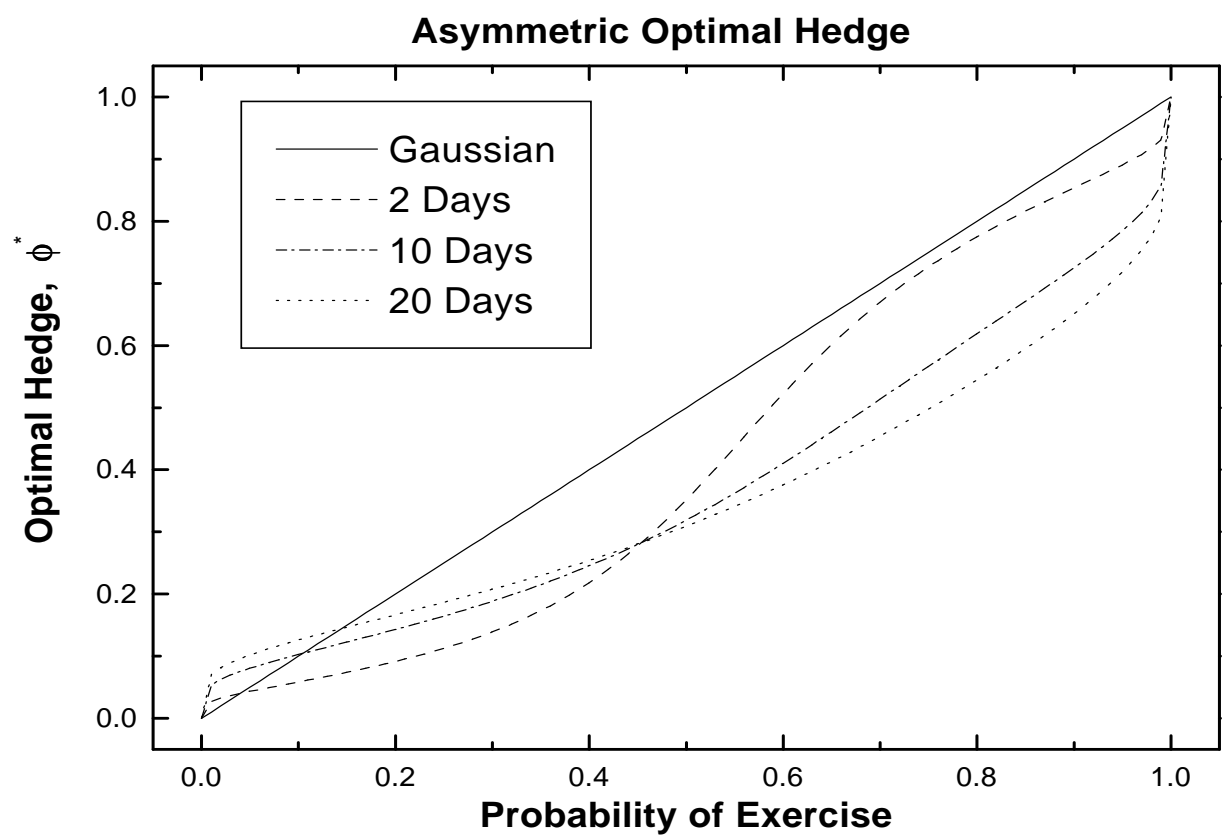

Figure 6: Solution to Eq. (3.20) with $\alpha=1.2, \lambda_{-}=1 / 122$ and $\lambda_{+}=1 / 41$. 